\title{
Characterization of Ice Roughness Variations in Scaled Glaze Icing Conditions
}

\author{
Stephen T. McClain ${ }^{1}$ \\ Baylor University, Waco, TX, 76798-73 \\ Mario Vargas ${ }^{2}$ \\ NASA Glenn Research Center, Cleveland, OH 44135 \\ and \\ Jen-Ching Tsao ${ }^{3}$ \\ Ohio Aerospace Institute, Cleveland, OH, 44142
}

\begin{abstract}
Because of the significant influence of surface tension in governing the stability and breakdown of the liquid film in flooded stagnation regions of airfoils exposed to glaze icing conditions, the Weber number is expected to be a significant parameter governing the formation and evolution of ice roughness. To investigate the influence of the Weber number on roughness formation, $53.3-\mathrm{cm}(21-\mathrm{in}$.$) and 182.9-\mathrm{cm}(72-\mathrm{in}$.$) NACA 0012$ airfoils were exposed to flow conditions with essentially the same Weber number and varying stagnation collection efficiency to illuminate similarities of the ice roughness created on the different airfoils. The airfoils were exposed to icing conditions in the Icing Research Tunnel (IRT) at the NASA Glenn Research Center. Following exposure to the icing event, the airfoils were then scanned using a ROMER Absolute Arm scanning system. The resulting point clouds were then analyzed using the self-organizing map approach of McClain and Kreeger (2013) to determine the spatial roughness variations along the surfaces of the iced airfoils. The roughness characteristics on each airfoil were then compared using the relative geometries of the airfoil. The results indicate that features of the ice shape and roughness such as glazeice plateau limits and maximum airfoil roughness were captured well by Weber number and collection efficiency scaling of glaze icing conditions. However, secondary ice roughness features relating the instability and waviness of the liquid film on the glaze-ice plateau surface are scaled based on physics that were not captured by the local collection efficiency variations.
\end{abstract}

\section{Nomenclature}

$\begin{array}{ll}A_{c} & =\text { accumulation parameter } \\ \mathrm{AOA} & =\text { angle of attack } \\ \mathbf{b} & =\text { codebook vectors } \\ h(i, j) & =\text { neighborhood function of } i \text { to } j \text { codebook vectors } \\ j & =\text { codebook vector index } \\ \mathrm{LWC} & =\text { liquid water content }\left[\mathrm{gm} / \mathrm{m}^{3}\right] \\ M & =\text { number of codebook vectors } \\ \mathrm{MVD} & =\text { median volumetric diameter }[\mu \mathrm{m}] \\ N & =\text { airfoil or mean ice shape surface normal coordinate direction } \\ n_{0} & =\text { stagnation point freezing fraction } \\ R^{d} & =\text { high-dimensional data space }\end{array}$

\footnotetext{
${ }^{1}$ Associate Professor, Department of Mechanical Engineering, One Bear Place \#97356, and AIAA Senior Member.

${ }^{2}$ Aerospace Engineer, Icing Branch, 21000 Brookpark Rd., AIAA Associate Fellow.

${ }^{3}$ Principal Research Scientist, Icing Branch, 21000 Brookpark Rd., MS 11-2, AIAA Associate Fellow.
} 


$\begin{array}{ll}R M H & =\text { roughness maximum height } \\ R_{q} & =\text { the root-mean-square or "standard deviation" roughness height } \\ r_{a} & =\text { leading edge radius of curvature } \\ \mathrm{SOM} & =\text { Self-Organizing Map } \\ S & =\text { airfoil or mean ice shape surface tangential coordinate direction } \\ \mathrm{SEE} & =\text { Standard error of the estimate for regression } \\ \mathrm{We} & =\text { Weber number } \\ x & =\text { element of data set } \\ \alpha & =\text { local direction angle of manifold through a codebook vector } \\ \beta & =\text { collection efficiency } \\ \beta_{0} & =\text { stagnation collection efficiency } \\ \gamma & =\text { direction angle of surface point relative to manifold direction through winning codebook vector }\end{array}$

\section{Introduction}

T ASER scanning of ice shapes has revolutionized the process of ice shape and ice roughness characterization. LPrevious laser-scanning based studies of ice roughness spatial and temporal evolution, such as McClain et al. [1] and McClain et al. [2], have focused on replicating the geometries and conditions used in historical roughness studies [3-5] performed in the Icing Research Tunnel (IRT) at NASA Glenn Research Center in Cleveland, OH. That is, the historical and recent laser-based ice roughness investigations were performed using $53.3-\mathrm{cm}$ (21-in.) NACA 0012 airfoils in low-speed highly-glazed icing conditions. The recent laser-scan based investigations have illuminated many important aspects of roughness temporal and spatial evolution that were previously unknown; however, the implications of the roughness studies related to large commercial aircraft wings and flight envelopes must be inferred using proper scaling of the results. While scaling large ice shape features from small geometries explored in the IRT has been a frequent research topic, proper scaling of roughness evolution physics has not received significant attention primarily because of the inability to quantify roughness before the maturation of laserscanning techniques in the IRT.

Scaling of icing roughness features, like scaling of full ice shapes, is complicated because the physics of the ice accretion includes multiple species (air and water), all three phases of water, and multiple phenomena, such as highshear and potentially turbulent freestream flow and a liquid film flow on the surface of the ice. Further, when supercooled water droplets impinge on an airfoil in glaze icing conditions, freezing is not immediate. As freezing takes place, a smooth ice layer is formed around the leading edge. At some chord-wise distance downstream from the stagnation line, there is a transition from smooth to rough ice zone. The surface runback water effects were believed to influence the shape of the accreted ice to some extent.

Recent ice-shape scaling studies [Ref. 6 and 7 for example] have further shown that acceptable glaze main iceshape similarity could be achieved by matching the stagnation collection efficiency $\left(\beta_{0}\right)$, the accumulation parameter $\left(A_{c}\right)$, the stagnation point freezing fraction $\left(n_{0}\right)$, and the Weber number $\left(W e_{L}\right)$, where

$$
\mathrm{We}_{L}=\frac{\rho_{w} U_{\infty}^{2} L}{\sigma}
$$

Based on the parameters being suspected as relevant to proper scaling, the Reynolds number is defined as

$$
\operatorname{Re}_{L}=\frac{\rho_{\text {air }} U_{\infty} L}{\mu_{\text {air }}}
$$

and the Stokes number is defined as

$$
S k=\frac{\rho_{w} U_{\infty} M V D^{2}}{18 \mu_{\text {air }} L}
$$

When the length scale employed in the Stokes number definition is the leading edge radius of the airfoil, the result is commonly referred to in the icing literature as the droplet inertial parameter, $K$ : 


$$
K=\frac{\rho_{w} U_{\infty} M V D^{2}}{18 \mu_{\text {air }} r_{a}}
$$

To maintain droplet trajectory similarity, the Langmuir and Blodgett [8] stagnation collection efficiency, $\beta_{0}$, is employed where

$$
\beta_{0}=\frac{1.4\left(K_{0}-\frac{1}{8}\right)}{1+1.4\left(K_{0}-\frac{1}{8}\right)}
$$

In Eq. (5), $K_{0}$ is the modified inertial parameter of Langmuir and Blodgett [8], defined as

$$
K_{0}=\frac{1}{8}+\frac{\lambda}{\lambda_{S k}}\left(K-\frac{1}{8}\right)
$$

where the parameter $\lambda / \lambda_{S k}$ is the droplet range parameter and is defined as a function of the droplet Reynolds number, $\operatorname{Re}_{\delta}=\rho V M V D / \mu$, as

$$
\frac{\lambda}{\lambda_{S k}}=\frac{1}{0.8388+0.001483 \mathrm{Re}_{\delta}+0.01847 \sqrt{\mathrm{Re}_{\delta}}}
$$

While the Reynolds number is relevant to the process of ice shape scaling, the Weber number and the stagnation collection efficiency are expected to be the dominant dimensionless parameters in scaling glaze ice accretions.

As part of an on-going effort to develop appropriate ice-accretion scaling methods for super-cooled large droplets (SLD) in glaze icing conditions, tests were conducted to obtain additional ice roughness data in both Appendix C (freezing drizzle, [9]) and SLD (freezing rain, [10]) regimes to assess how well the ice roughness features would be simulated in NACA 0012 airfoils of different chord sizes when the aforementioned constant $W e_{L}$ scaling method is applied. For this study, the models used were un-swept NACA 0012 airfoil sections mounted vertically in the center of the test section of the IRT at the NASA Glenn Research Center. Reference tests were performed with a 6-ft span, 72-in.-chord aluminum model, and the scale tests were performed with a 6-ft span, 21in. chord aluminum model, as shown in Fig. 1 (a) \& (b).

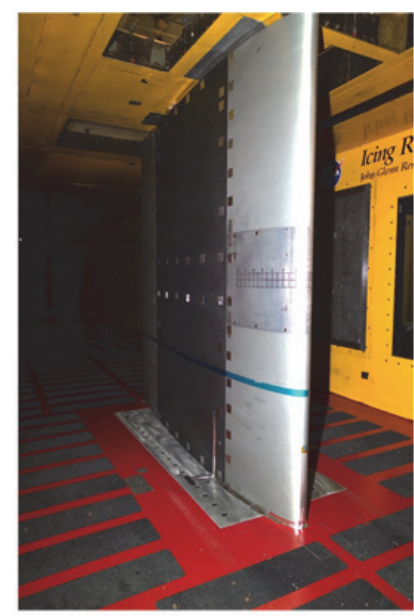

(a) 72-in-Chord Full-Span Model

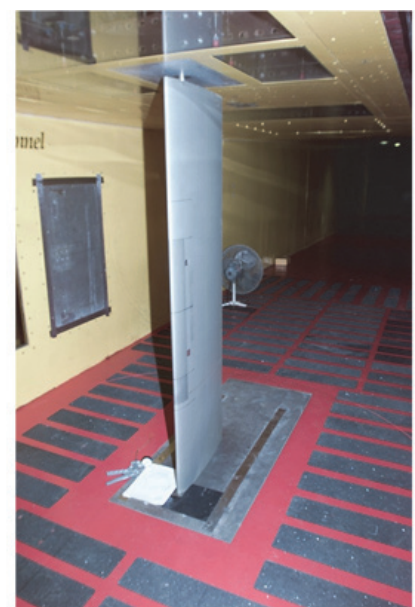

(b) 21-in-Chord Full-Span Models

Figure 1. NACA 0012 Models Installed in IRT Test Section: (a) the 72-in.-Chord Model and (b) the 21-in.-Chord Model 


\section{Methodology}

To investigate the roughness of ice shapes on scaled airfoils, the shapes were 1) created, 2) measured, and then 3) characterized using the SOM approach of McClain and Kreeger [11]. The following sections describe each of the ice roughness generation and characterization steps.

\section{A. Ice Shape Generation}

All of the experimental measurements were performed in the IRT. Straight 72-in. (182.9-cm) and 21-in. (53.3$\mathrm{cm})$ chord NACA 0012 airfoils, which spanned the entire 72-in. $(182.9 \mathrm{~cm})$ vertical dimension of the test section, were used for the study. For the investigation, the tests completed on the 72 -in. airfoil are referred to as the "reference tests", and the tests completed on the 21 -in. airfoils are referred as the "scaled tests." For all tests, the test airfoil AOA was set to $0^{\circ}$.

Table 1 presents the freestream total temperature $\left(T_{\text {total }}\right)$, the freestream velocity, the median volumetric diameter, the liquid water content, the accumulation parameter, the chord-based Reynolds number, the stagnation collection efficiency, and the Weber number based on the twice the leading edge radius as the length scale. The accumulation parameter is a derived quantity calculated using

$$
A_{c}=\frac{L W C \cdot V \cdot \Delta t_{s}}{2 \cdot r_{a} \cdot \rho_{\text {ice }}}
$$

Where $r_{a}$ is the leading-edge radius of curvature of the NACA 0012 airfoil, and $\rho_{\text {ice }}$ is the density of ice at the freestream temperature. As shown in Table 1, the primary experimental dimensions varied during the reference tests were the MVD and the resulting stagnation collection efficiency. For the scaled tests, the MVD and the accretion time, $\Delta t_{s}$, were varied to match the accumulation parameter $\left(A_{c}\right)$, the Weber number using twice the leading edge radius as the length scale, and the stagnation point collection efficiency. For all of the cases used in this study, the stagnation freezing fraction, $n_{0}$, was set to 0.25 . Table 1 demonstrates that while the Reynolds number changed between the reference to the scaled cases, the Weber numbers match. Further for each reference condition on the 72-in. airfoil, a scaled condition was created to match both Weber number and stagnation point collection efficiency.

Table 1. Summary of Test Parameters for Roughness Scaling Investigation

\begin{tabular}{|c|c|c|c|c|c|c|c|c|c|c|}
\hline $\begin{array}{c}\text { Case } \\
\text { Number }\end{array}$ & $\begin{array}{c}\text { Chord } \\
\mathbf{c m}(\mathbf{i n} .)\end{array}$ & $\begin{array}{c}\boldsymbol{T}_{\text {total }} \\
\left({ }^{\mathbf{C}} \mathbf{C}\right)\end{array}$ & $\begin{array}{c}\mathbf{V} \\
(\mathbf{m} / \mathbf{s})\end{array}$ & $\begin{array}{c}\mathbf{M V D} \\
(\boldsymbol{\mu m})\end{array}$ & $\begin{array}{c}\mathbf{L W C} \\
\left(\mathbf{g m} / \mathbf{m}^{\mathbf{3}}\right)\end{array}$ & $\begin{array}{c}\Delta \boldsymbol{t}_{\boldsymbol{s}} \\
(\mathbf{s e c})\end{array}$ & $\boldsymbol{A}_{\boldsymbol{c}}$ & $\mathbf{R e}_{\mathbf{c}} \times \mathbf{1 0}^{-\mathbf{6}}$ & $\boldsymbol{\beta}_{\boldsymbol{0}}$ & $\mathbf{W e}_{\mathbf{d}} \times \mathbf{1 0}^{-\mathbf{6}}$ \\
\hline 041514.01 & $183(72)$ & -4.7 & 51.5 & 50.0 & 0.75 & 477.1 & 0.348 & 6.68 & 0.710 & 2.36 \\
\hline 041514.02 & $183(72)$ & -5.5 & 51.5 & 100.0 & 0.75 & 477.1 & 0.348 & 6.68 & 0.859 & 2.36 \\
\hline 041514.03 & $183(72)$ & -5.7 & 51.5 & 150.0 & 0.75 & 477.1 & 0.348 & 6.68 & 0.911 & 2.36 \\
\hline 041514.04 & $183(72)$ & -5.8 & 51.5 & 174.0 & 0.75 & 477.1 & 0.348 & 6.68 & 0.925 & 2.36 \\
\hline 032814.05 & $53.3(21)$ & -2.9 & 95.3 & 18.2 & 0.90 & 62.6 & 0.348 & 3.61 & 0.710 & 2.36 \\
\hline 032814.02 & $53.3(21)$ & -4.0 & 95.3 & 35.8 & 1.00 & 56.4 & 0.348 & 3.61 & 0.859 & 2.36 \\
\hline 032814.03 & $53.3(21)$ & -2.3 & 95.3 & 53.2 & 0.58 & 97.2 & 0.348 & 3.61 & 0.911 & 2.36 \\
\hline 032814.04 & $53.3(21)$ & -2.7 & 95.3 & 61.5 & 0.65 & 86.7 & 0.348 & 3.61 & 0.925 & 2.36 \\
\hline
\end{tabular}

For each test, the airspeed and freestream total temperature were set and the spray bar air and water pressures were selected to provide the appropriate LWC, MVD, and freezing fraction. A thermocouple embedded in the NACA 0012 airfoil was used to determine when the airfoil had reached thermal equilibrium with the flow. Once the thermocouple reported the static temperature of the flow indicating that it was in thermal equilibrium with the flow, the spray bars were actuated and closed after the predetermined spray time.

\section{B. Ice Shape Measurement}

Following the completion of the icing spray, the wind tunnel velocity was reduced to approximately 10 knots (5 $\mathrm{m} / \mathrm{s}$ ) while keeping the static temperature around $-4{ }^{\circ} \mathrm{C}$ to avoid thawing of the ice shape. The iced airfoil was painted using an alcohol-based titanium dioxide paint. A ROMER Absolute Arm laser scanning system was placed in the IRT test section upstream of the airfoil. A scan was then made of the leading 120-150 mm of the airfoil leading edge (in the streamwise direction). The scans were approximately $100-\mathrm{cm}$ wide (in the spanwise direction) and were performed at the center-span location of the airfoil. 


\section{SOM Roughness and Thickness Evaluation}

The surface point clouds were then processed using the Self-Organizing Map (SOM) approach developed by McClain and Kreeger [11]. The self-organizing map, or sometimes referred to as a Kohonen Map, is a clustering method for the detection of non-linear manifolds, which may be curves or surfaces, in multi-dimensional space [12]. SOMs depend on the use of codebook vectors, $\mathbf{b}$, which may also be called codebook points or neurons, to represent clumps of data. Following convergence of the SOM method, each codebook vector will be located at the spatial centroid of the clump of data that it represents. In its simplest essence, self-organizing maps are employed to capture trends of large data sets by representing those large data sets by a relatively small set of codebook vectors. When applied to an ice shape, the intent of performing the SOM is to extract the "form" of the ice shape from the surface variations. For detailed information on self-organizing maps and their application for iced airfoil description and roughness evaluation, please consult Refs. [1] and [2].

When applied to an iced airfoil point cloud without sweep or significant spanwise shape changes, the SOM is expected to identify a curve in the Chord-Chord Normal plane (or x-y plane when the spanwise axis is placed in the z-direction), which represents the mean shape of the rough airfoil. The nature of the SOM method and the positioning of the codebook vectors along a "daisy-chain" enable a statistical evaluation of iced airfoil surface roughness. Since the "clumps" of points are distributed about the codebook vectors, the deviations of the point measurements in the clumps can be used to evaluate the coverage statistics and uncertainty of the codebook vector representation.

Figure 2 shows a single surface measurement, $\mathbf{x}^{\mathrm{j}}$, and its closest codebook vector $\mathbf{b}^{\mathbf{n}}$. The two neighboring codebook vectors along the daisy-chain of codebook vectors representing the manifold are also shown. In the approach used for this study, the manifold is assumed to be a first-order manifold in two-dimensional space with the characteristic that at each codebook vector the local slope of the manifold is equal to the central finite-difference evaluated using the two closest surrounding codebook vectors. The approach used assumes that all deviations from the manifold are normal to the manifold. That is, the deviation of a surface measurement normal to the line through the codebook vector with the local slope set by the neighboring codebook vectors is considered the "height" of the surface point above or below the local manifold.

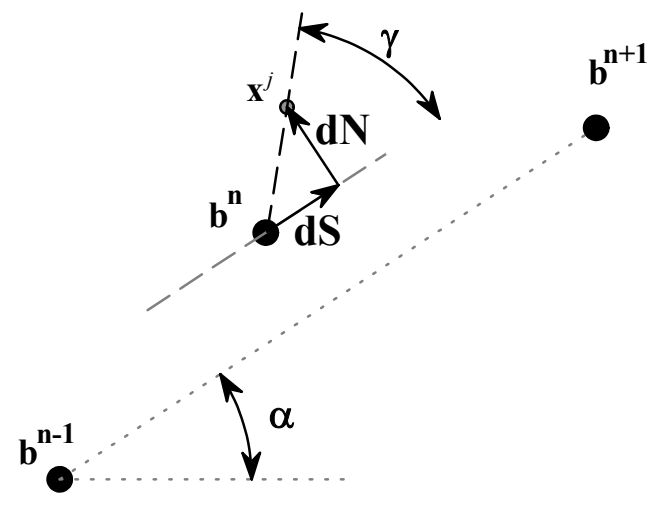

Figure 2. Metrics of Local Point about a Codebook Vector [9]

To calculate the local height of any point, $\mathbf{x}^{\mathrm{j}}$, relative to the manifold, the two neighboring codebook vectors are first used to calculate the direction of the manifold through $\mathbf{b}^{\mathbf{n}}$ using

$$
\alpha_{\mathbf{b}^{\mathbf{n}}}=\arctan \left[\frac{y_{\mathbf{b}^{\mathrm{n}+1}}-y_{\mathbf{b}^{\mathrm{n}-1}}}{x_{\mathbf{b}^{\mathrm{n}+1}}-x_{\mathbf{b}^{\mathrm{n}-1}}}\right]
$$

The direction of the $\mathbf{x}^{\mathrm{j}}$ point from its closest codebook vector relative to the line through the codebook vector with the direction $\alpha$ is then found using

$$
\gamma_{\mathbf{x}^{j}}=\arctan \left[\frac{y_{\mathbf{x}^{j}}-y_{\mathbf{b}^{\mathbf{n}}}}{x_{\mathbf{x}^{j}}-x_{\mathbf{b}^{\mathbf{n}}}}\right]-\alpha_{\mathbf{b}^{\mathbf{n}}}
$$


The normal height the $\mathbf{x}^{\mathrm{j}}$ point from the line through its closest codebook vector is then determined using

$$
N_{\mathbf{x}^{j}}=\left[\left(x_{\mathbf{x}^{j}}-x_{\mathbf{b}^{\mathbf{n}}}\right)^{2}+\left(y_{\mathbf{x}^{j}}-y_{\mathbf{b}^{\mathbf{n}}}\right)^{2}\right]^{1} \sin \left(\gamma_{\mathbf{x}^{j}}\right)
$$

The normal height of all the points related to an individual codebook vector may then be used to calculate statistics such as those commonly used to calculate traditional roughness parameters. For example, the root-mean-square roughness height for a rough surface is traditionally described as

$$
R_{q}=\left[\frac{1}{J} \sum_{j=1}^{J}\left(y_{j}-\bar{y}\right)^{2}\right]^{\frac{1}{2}}
$$

Based on the SOM-manifold description used here, the root-mean-square roughness height is calculated at each codebook vector as

$$
R_{q}=\left[\frac{1}{J} \sum_{j=1}^{J} N_{\mathbf{x}^{j}}{ }^{2}\right]^{\frac{1}{2}}
$$

In some roughness studies, the $99.9 \%$ roughness maximum height (RMH) based on a Gaussian distribution is calculated using 3.09 times the root-mean roughness height.

$$
R M H_{\mathbf{b}^{\mathbf{n}}}=3.09 R_{q}=3.09\left[\frac{1}{J} \sum_{j=1}^{J} N_{\mathbf{x}^{j}}\right]^{\frac{1}{2}}
$$

Where $J$ is the number of surface points for which $\mathbf{b}^{\mathbf{n}}$ is the winning (closest) codebook vector. The RMH is the local 99.9\%-maximum roughness height and is evaluated at each codebook vector based on the local or neighborhood statistics. The RMH will vary along the surface arc length. While the RMH is not a universal roughness descriptor, the RMH value is important for this study. Since the RMH represents a Gaussian prediction of the $99.9 \%$ maximum distance from the mean elevation to the tallest peaks in a data set, the RMH is a reasonable tool to compare the statistical results to the morphological descriptors such as roughness element diameter and height used in the historical roughness studies.

To evaluate the distance along the manifold representing the mean ice shape, a discrete arc-length approach is taken. That is, at one end of the daisy chain, the length of the arc is set to zero or a known value from the stagnation point on the airfoil. The position of the next codebook vector is then evaluated as the straight-line distance between the two codebook vectors as demonstrated in Eq. (15).

$$
S_{\mathbf{b}^{\mathbf{n}}}=S_{\mathbf{b}^{\mathbf{n}-1}}+\left[\left(x_{\mathbf{b}^{\mathbf{n}}}-x_{\mathbf{b}^{\mathbf{n}-1}}\right)^{2}+\left(y_{\mathbf{b}^{\mathbf{n}}}-y_{\mathbf{b}^{\mathbf{n}-1}}\right)^{2}\right]^{\frac{1}{2}}
$$

Once the surface distance coordinate of each codebook vector is determined, the surface distance coordinate of each point cloud measurement may be evaluated based on the location of the surface point's winning codebook vector. Revisiting Figure 2, once the angle of the $\mathbf{x}^{\mathrm{j}}$ point with respect to the surface manifold through its winning codebook vector $(\gamma)$ is known, the surface projection along the manifold is found using

$$
S_{\mathbf{x}^{j}}=S_{\mathbf{b}^{\mathbf{n}}}+d S_{x^{j}}=S_{\mathbf{b}^{\mathbf{n}}}+\left[\left(x_{\mathbf{x}^{j}}-x_{\mathbf{b}^{\mathbf{n}}}\right)^{2}+\left(y_{\mathbf{x}^{j}}-y_{\mathbf{b}^{\mathbf{n}}}\right)^{2}\right]^{\frac{1}{2}} \cos \left(\gamma_{\mathbf{x}^{j}}\right)
$$


The ice thickness relative to the clean airfoil shape, $N O_{x}^{j}$, is calculated in a fashion very similar to the ice roughness value calculated in Eq. (11) with the exception of instead of using the codebook vector as the surface reference, the original airfoil design coordinates are used. More details regarding the ice thickness evaluation may be found in McClain [13].

\section{Results and Discussion}

Because of the size and nature of the resulting point clouds from the reference and scaled ice shapes, the results are presented and discussed in four steps. First, a visual comparison of an example set reference and scaled conditions is presented to demonstrate the substantial differences of the measured point clouds in unscaled coordinates. Second, the results of the roughness and thickness measurements are presented in unscaled coordinates to demonstrate the effects of the resulting stagnation collection efficiency on the resulting ice roughness and thickness variations, Third, the roughness and thickness results are presented and compared in scaled coordinates for cases with the same stagnation point collection efficiency. Finally, an approach to scale all of resulting roughness and thickness measurements for generality along the airfoil surface is presented.

Figure 3 presents a side-by-side comparison of the 041514.01 point cloud to the 032814.05 point cloud, which as noted in Table 1 was created to match the Weber number, stagnation point collection efficiency, freezing fraction, and accumulation parameter of the 041514.01 case. While recognizing that the color maps for the point clouds have different ranges, Figure 3 demonstrates that the roughness elements on the 041514.01 case are much taller and wider than the roughness elements on the 032814.05 case.

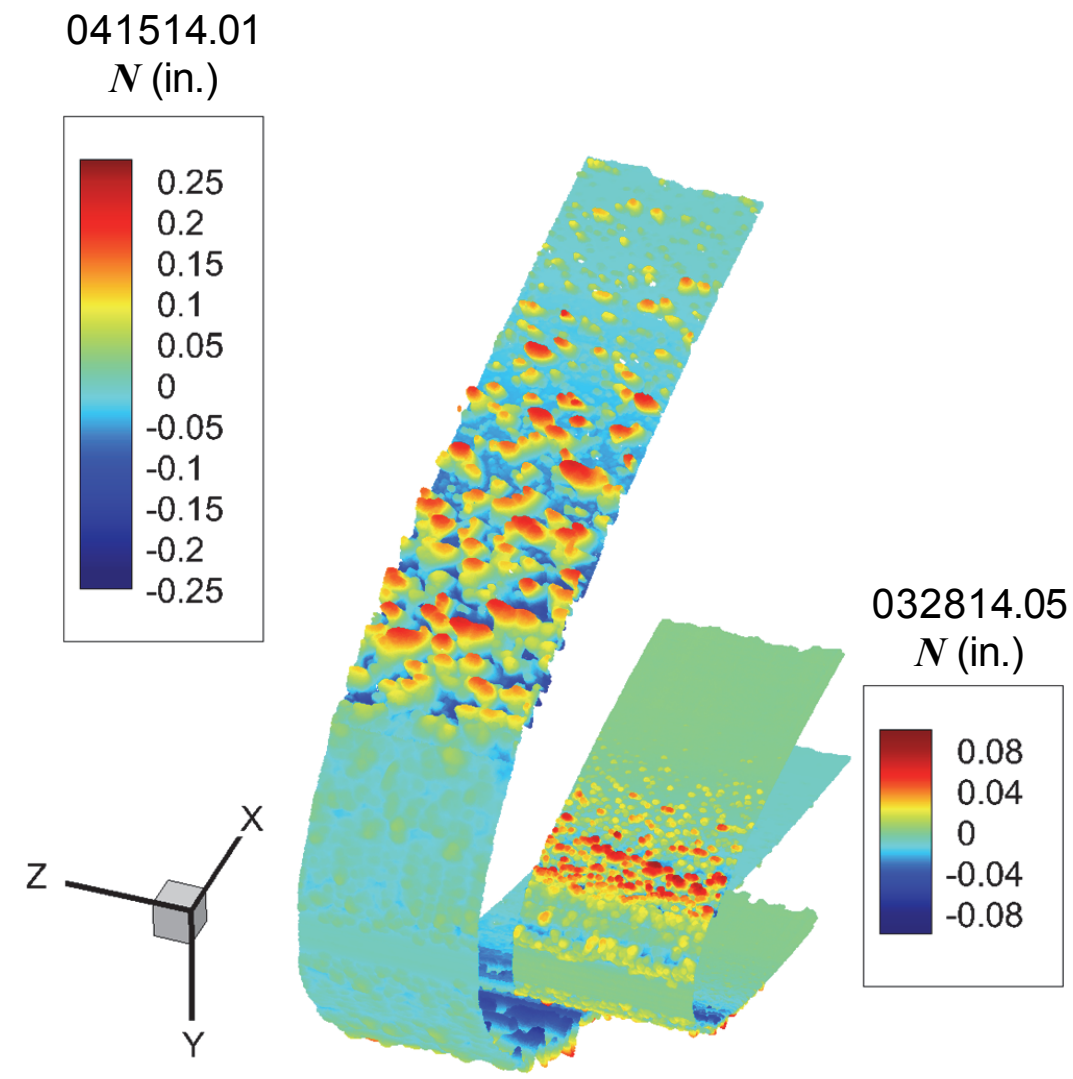

Figure 3. Comparison of Two Scaled Point Clouds with Same Weber Number and Stagnation Point Collection Efficiency

Figure 4 presents the measured variations in RMS roughness at each SOM codebook vector versus the surface distance from the airfoil stagnation point for the reference and scaled airfoil cases. Figure 5 presents the measured average ice thickness at each SOM codebook vector versus the surface distance from the airfoil stagnation point for the reference and scaled airfoil cases. In Figs. 4 and 5, the subfigures (a) are for the reference case and the 
subfigures (b) are for the scaled cases. Further, in Figs. 4 and 5, the different stagnation collection efficiencies are color coded in that the lowest value of $\beta_{0}=0.710$ are red, while the highest value of $\beta_{0}=0.925$ are gray.

Figs 4 and 5 demonstrate significant variations in the ice roughness and ice thickness with the different stagnation point collection efficiencies studied. All of the roughness profiles exhibit three distinct regions: 1) a smooth stagnation point region, 2) a region of isolated roughness elements on an ice plateau upstream of the plateau break down, and 3) the region of very tall roughness elements downstream of the ice plateau which decays in height in the flow direction. While all of the profiles exhibit the three distinct regions, Fig. 4 demonstrates that the heights of the isolated elements on the ice plateau are much larger for the 21-in. airfoil cases relative to the maximum roughness heights on the airfoil. Fig. 4 further demonstrates the effect of the stagnation point collection efficiency on the "fullness" of the roughness region. As the MVD and $\beta_{0}$ decrease, the maximum RMS roughness height decreases and the decay of the roughness values occurs more rapidly in the increasing surface distance direction. The stagnation point and ice plateau thickness values show much less variation with the changing MVD and $\beta_{0}$ values in Fig. 5. However, Fig. 5 does demonstrate that the decreasing MVD and $\beta_{0}$ values affect the decay of the average thickness in much the same way as the more rapid decrease in roughness values along the airfoil surface with decreasing MVD and $\beta_{0}$ values.

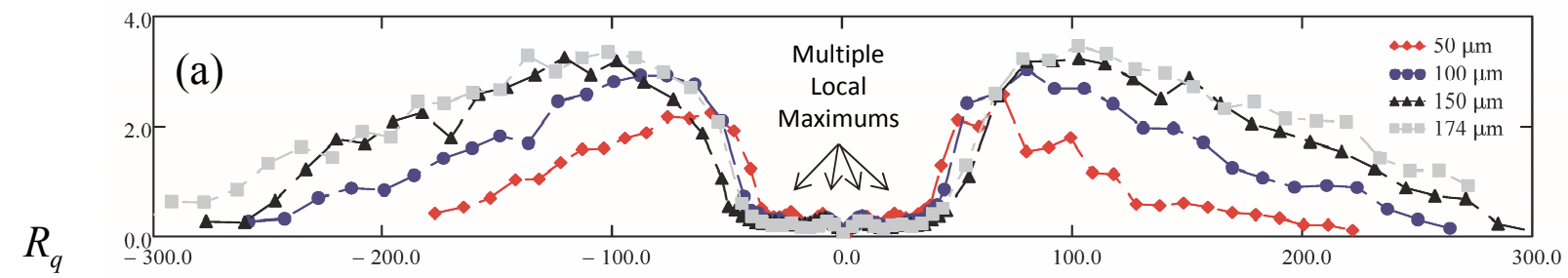

$(\mathrm{mm})$

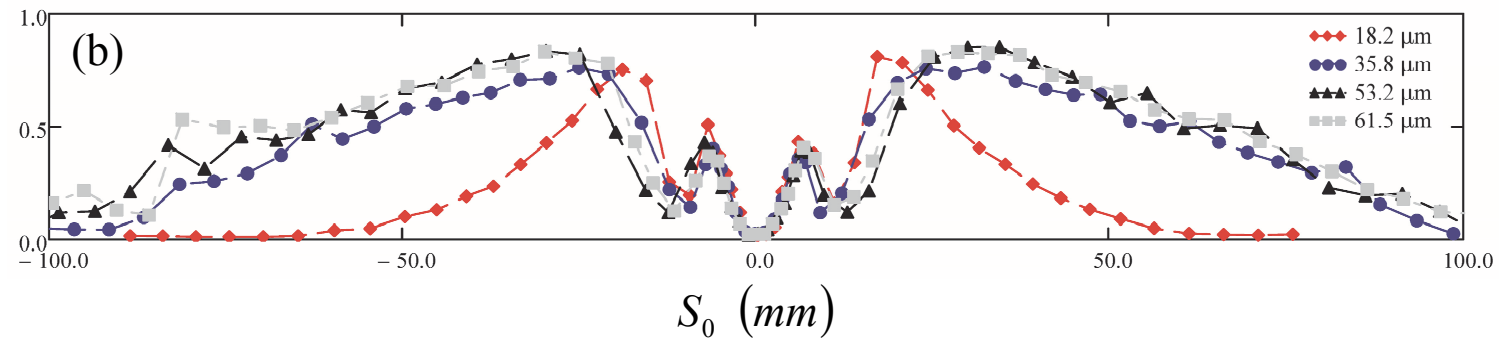

Figure 4. Measured RMS Roughness Heights along the Surface of the Airfoils: (a) Reference Condition with 72 in. Chord, (b) Scaled Condition with 21-in. Chord

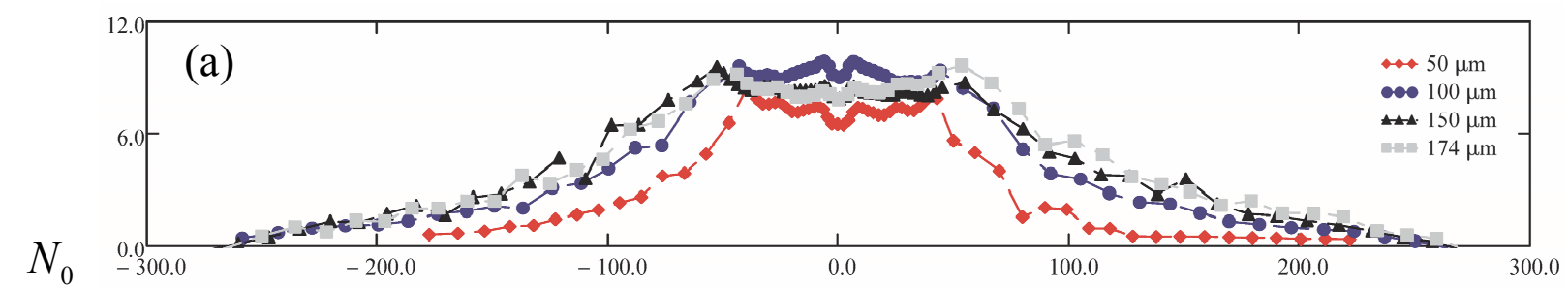

$(\mathrm{mm})$

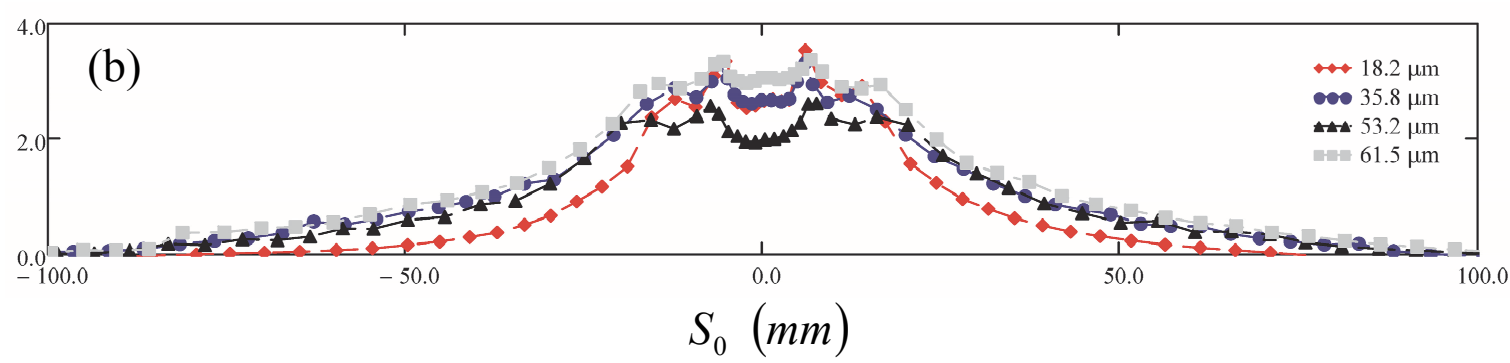

Figure 5. Measured Ice Thickness along the Surface of the Airfoils: (a) Reference Condition with 72-in. Chord, (b) Scaled Condition with 21-in. Chord 
To illuminate the geometric similarities in the roughness results, Figure 6 presents the relative roughness height variations along the surfaces of the reference and scaled airfoils. While either airfoil chord or leading-edge radius could be used to normalize the roughness values for the relative comparison, the chord is used to scale both the roughness and the surface distance from the stagnation point. Each subfigure of Figure 6 represents a comparison of the reference and scaled conditions for a given value of $\beta_{0}$. Figure 6 demonstrates that for each value of stagnation point collection efficiency, the relative roughness profiles of the scaled conditions track the reference cases very well except for the isolated roughness elements on the surface of the glaze ice plateau.

Similar to Figure 6, Figure 7 presents the relative ice thickness variations versus the scaled surface distance from the stagnation point. Just as with the roughness comparison, the most significant differences appear in the glaze ice plateau region. Outside of the glaze ice plateau, the relative thickness variations are very similar for each value of $\beta_{0}$.

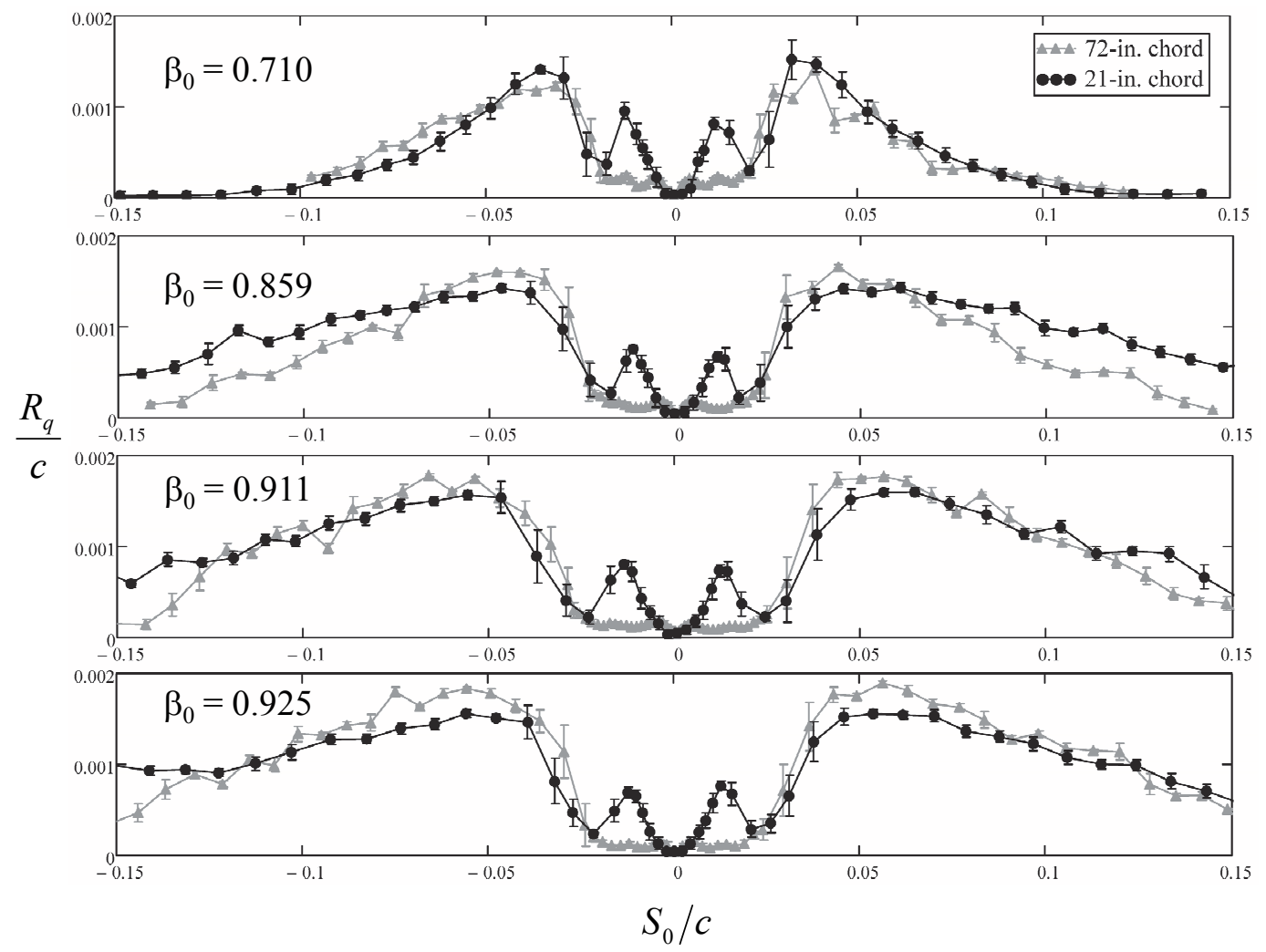

Figure 6. Relative Roughness versus Surface Distance Profiles Grouped by Collection Efficiency

While Figs. 6 and 7 show geometric similarity between both roughness and thickness for airfoils with matched Weber number, stagnation collection efficiency, and accumulation parameter, a method to collapse all of the reference and scaled cases was also investigated. For cases when liquid-film dynamics are negligible compared to the particle impact and collection physics, employing a scaling that correctly scales geometry with the local collection efficiency should collapse all of the cases studied. To further scale the roughness and thickness geometry, the theoretical Rime-ice stagnation point thickness based on accumulation parameter and assuming a freezing fraction of $100 \%$ is found using

$$
N_{0, R}=2 r_{0} A_{c} \beta_{0}
$$

The scaled maximum roughness is then calculated by dividing the $99 \%$-Gaussian Roughness Maximum Height $(\mathrm{RMH})$ by the Rime-ice stagnation point thickness, as shown in Eq. (18).

$$
\frac{R M H}{N_{0, R}}=\frac{3.09 R_{q}}{2 r_{0} A_{c} \beta_{0}}
$$


The RMH values are preferred in Eq. (18) because McClain et al. [7] showed that the maximum roughness height was related to the glaze ice plateau thickness. Consequently, the scaled RMH values are expected to be of similar magnitude as the scaled thickness values. The scaled local thickness values are then calculated as

$$
\frac{\bar{N}_{0}}{N_{0, R}}=\frac{\bar{N}_{0}}{2 r_{0} A_{c} \beta_{0}}
$$

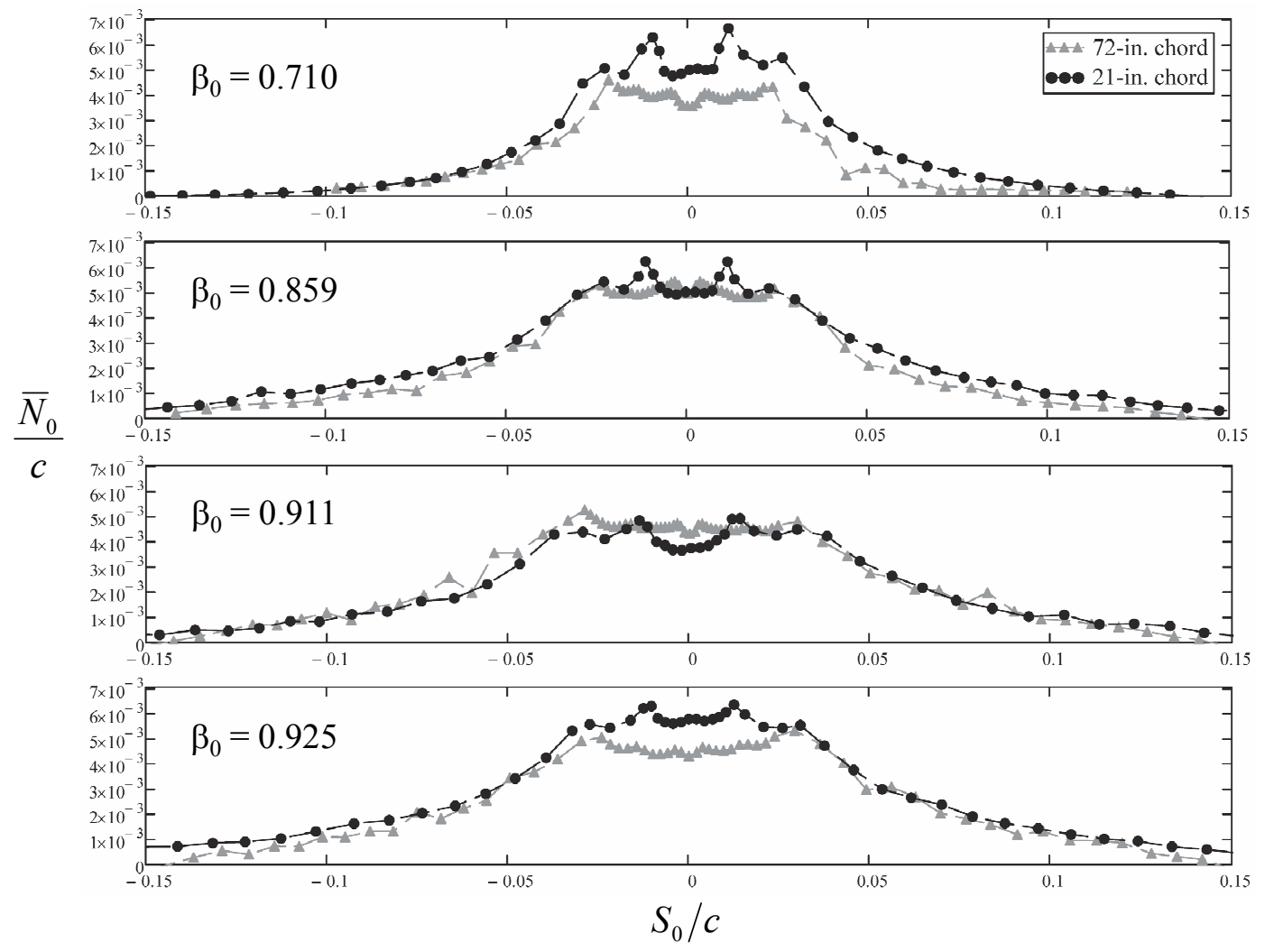

Figure 7. Relative Local Ice Thickness versus Surface Distance Profiles Grouped by Collection Efficiency

In Rime ice conditions, the relative ice thickness values would be expected to correlate with the local ice collection efficiency. While the stagnation point collection efficiency is readily computed using Eq. (5), calculating the local collection efficiency on a specific location on an airfoil is a difficult task. LEWICE performs the calculations by segmenting a cloud droplet distribution into many different diameter regimes and then predicting particle trajectories for each of the different regimes [14]. While determining the actual local surface collection efficiency requires a detailed process, the local collection efficiency may be estimated using the local surface viewing angle relative to the flow. Figure 8 shows an airfoil exposed to a flow at an angle of attack where the local collection efficiency, $\beta_{s}$, is to be determined at some point on the airfoil. For a non-zero angle of attack, the local collection efficiency may be estimated as

$$
\beta_{s} \approx \beta_{0} \cos \left(\alpha+\gamma_{s}\right)
$$

Because ice thickness is most expected to be correlated by the Rime-ice stagnation point thickness and the local collection efficiency, Figure 9 presents the scaled thickness variations first. Figure 9 presents the values of scaled thickness at each codebook vector versus $\left(1-\beta_{s}\right)$. The scaled thickness values were plotted versus $\left(1-\beta_{s}\right)$ so that increasing values along the abscissa represents movement away from the stagnation point of the airfoil. 


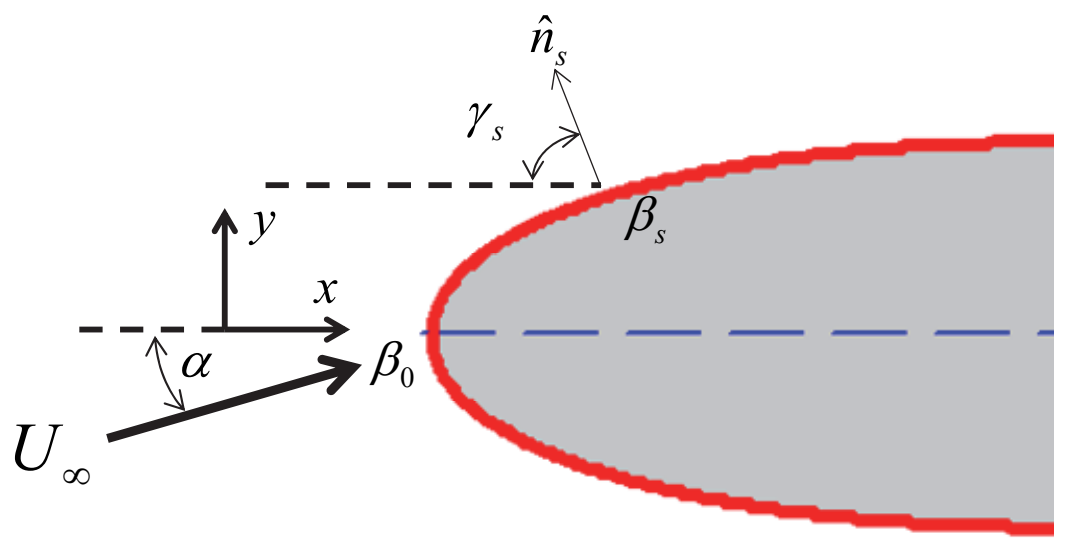

Figure 8. Geometric Evaluation of Local Surface Collection Efficiency

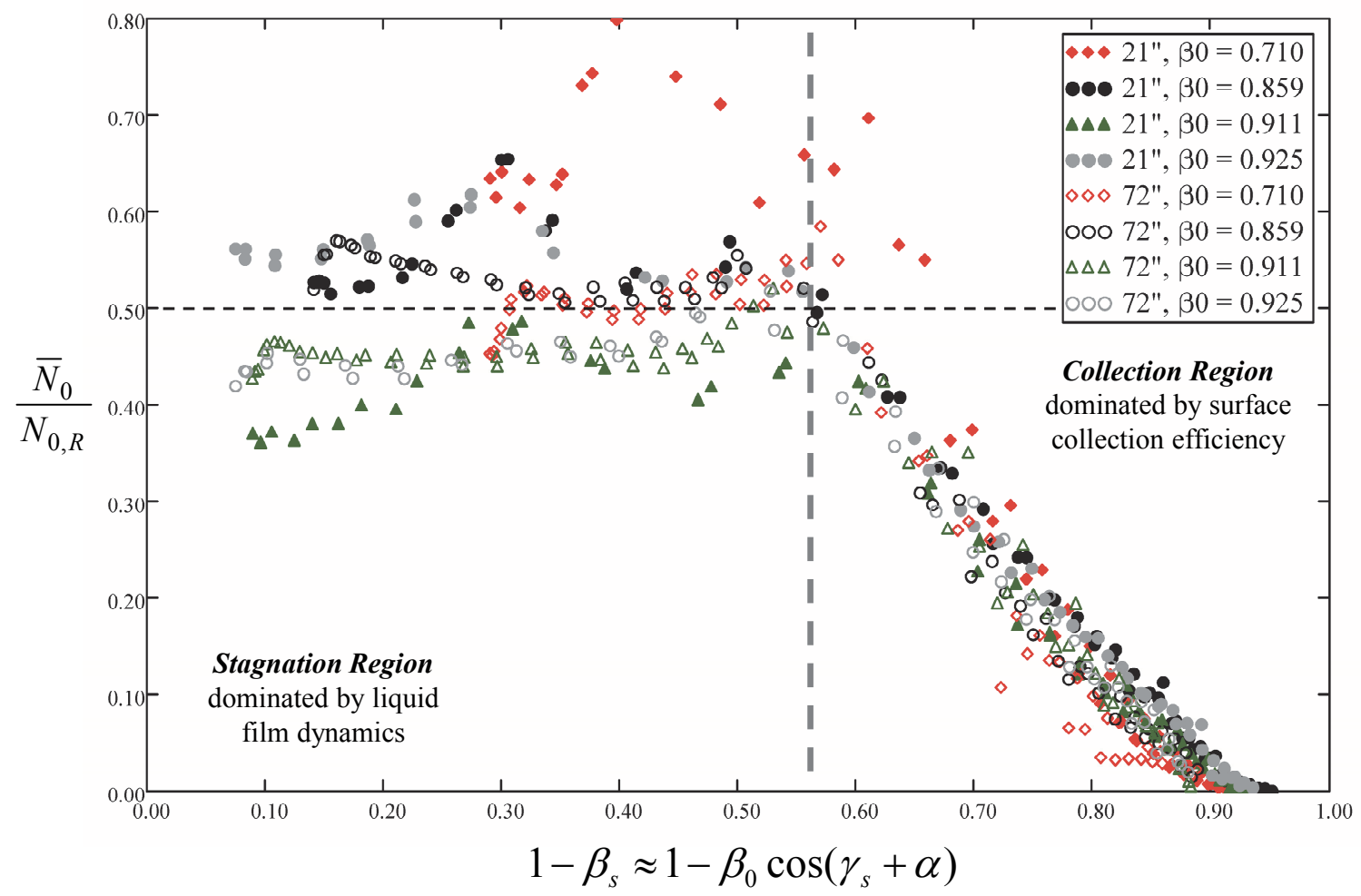

Figure 9. Scaled Ice Thickness versus Estimated Local Collection Efficiency

Figure 9 demonstrates that, while there is scatter in the scaled thickness data, two distinct regions of the scaled thickness profiles are evident. The first region which exists on the glaze ice plateau exhibits variations and significant experimental scatter but is not correlated with the local collection efficiency. The second region, which is on the right-hand side of the figure, is downstream of the glaze ice plateau where the scaled thickness values vary linearly with the estimated local collection efficiency. The presence of two distinct regions demonstrates that on the glaze ice plateau surface, the dominant physics that are occurring relate to the behavior of the liquid film as opposed to the droplet collection physics. Once downstream of the glaze-ice plateau and the location of the liquid film breakdown (as indicated by the vertical dashed line on Figure 9), droplet collection physics dominates the accumulation of ice on the airfoil surface.

Figure 10 presents the scaled maximum roughness variations at each codebook vector versus $\left(1-\beta_{s}\right)$. Like Figure 9 , Figure 10 exhibits two regions of behavior separated by the location of the glaze ice-plateau and liquid 
film breakdown, as indicated by the vertical dashed line. To the left of the vertical dashed line, liquid film dynamics dominate the physics and lead to isolated roughness elements on the glaze plateau surface. To the right of the vertical dashed line, collection efficiency dominates. Further, in the collection efficiency region, the scaled maximum roughness values increase downstream of the ice plateau until a maximum which is near the same average value of the scaled ice thickness on the plateau surface from Figure 9. Interestingly, in the collection efficiency region of the scaled maximum roughness height variation, the curves do not collapse as well as the scaled thickness trends. While reasons for the increased scatter are not isolated with this study, suspected causes are: 1) the difference in cloud droplet distributions created by the IRT spray bars at the smaller MVD values, 2) the difference in collection efficiencies as enhanced by the different size and nature of the roughness generated by each condition, and 3) the issue of calculating the maximum roughness height employing a Gaussian approach when the heights become more and more non-Gaussian further away from the glaze-ice plateau.

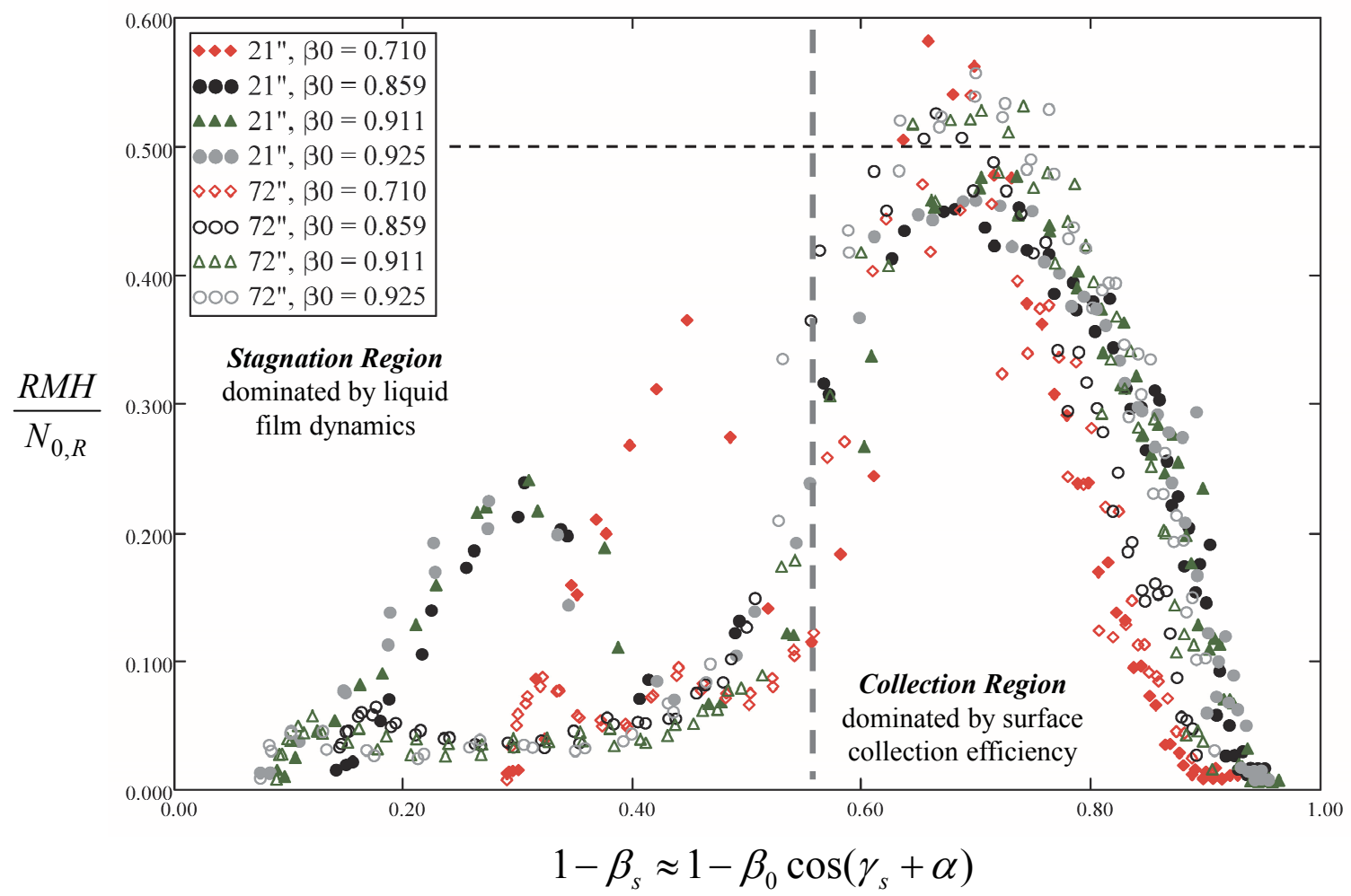

Figure 10. Scaled Maximum Roughness versus Estimated Local Collection Efficiency

To provide evidence of liquid-film dynamics influencing roughness formation in the stagnation region and ice plateau surface, Figure 11 presents the RMS roughness heights versus the surface distance from the stagnation points in un-scaled dimensions. Figure 11 demonstrates that all of the surfaces exhibit a region of isolated roughness of similar amplitude with a maximum between $7-9 \mathrm{~mm}$ from the stagnation point. The direct similarities in the first region of roughness elements indicates that flow and liquid film instabilities which are related to either $\mathrm{Re}_{x}$ or $\mathrm{We}_{x}$ are the dominant physics controlling the formation of the isolated roughness elements on the glaze ice plateau surface instead of droplet collection dynamics. Revisiting Figures 4 and 6 and inspecting the stagnation regions of the iced airfoil cases (on the glaze ice surface), several wavelengths or local maximums of roughness values are present for the 72-in. airfoil cases on either side of the stagnation point, while only one wavelength or local maximum is present on the 21-in. iced airfoil cases upstream of the location of the glaze-ice plateau on each side of the stagnation point. 


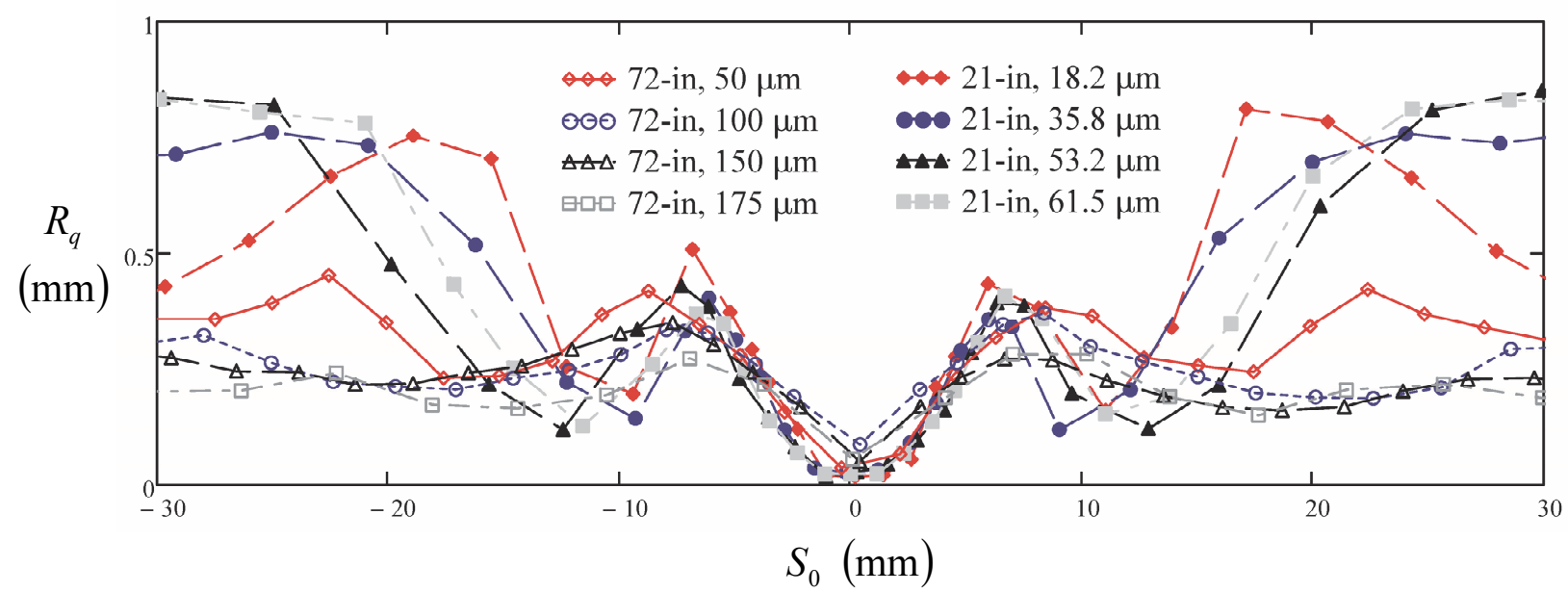

Figure 11. Unscaled RMS Roughness Variations near the Stagnation Region for all Airfoils

The trends exhibited in Figs. 9-11 provide substantial evidence for prediction of glaze ice roughness spatial evolution based on appropriate scaling arguments. When the results are further combined with the results of McClain et al. [6] for supercooled large droplet icing and McClain et al. [7] for freezing drizzle icing, which focus on the temporal development of the airfoil maximum roughness heights, anticipating a correlation for the full temporal and spatial development of both thickness and ice roughness in short-duration glaze icing conditions is reasonable. However, several questions remain unanswered regarding glaze ice roughness evolution. First, all of the scaling cases were performed for a fixed accumulation parameter $\left(A_{c}=0.348\right)$ and a fixed stagnation point freezing fraction $(n=0.25)$. More scaling cases at different accumulation parameters and freezing fractions are required to determine how the spatial variations in thickness and roughness are affected by these parameters. Further, all of the cases presented here are for straight, symmetric airfoils at zero angle of attack. More data are needed to fully describe the influence of the local collection efficiency with regards to swept, asymmetric, or pitched airfoils.

\section{Conclusions}

As part of a continued study on the spatial and temporal evolution of roughness on airfoils in glaze-icing conditions, the roughness variations on a 72-in. NACA 0012 airfoil were characterized using the Icing Research Tunnel at NASA Glenn Research Center. The roughness variations created on a 21-in. NACA 0012 exposed to conditions matching, accumulation parameter, the stagnation point freezing fraction, the Weber numbers and stagnation point collection efficiencies were also characterized. The ice roughness variations were characterized using the method of McClain and Kreeger [4], while the ice thickness variations were characterized using the method of McClain [8]. The primary conclusions of the study are:

1) Downstream of the glaze-ice plateau, ice thickness and ice roughness values scaled using the Rime-ice stagnation thickness match for scaled icing conditions based on Weber number and stagnation collection efficiency and vary with local collection efficiency,

2) On the glaze-ice surface, the liquid film physics are more important than collection physics, consequently the isolated roughness on the glaze surface are captured without scaling based on local Reynolds or local Weber numbers.

In the context of prior work regarding roughness temporal development, the current results suggest that while experimental scatter are evident in the data, correlations for the spatial and temporal evolution of roughness and glaze ice thickness are obtainable in short-duration icing events before substantial airfoil shape deformation occurs. Further, the results of the study indicate that outside of the glaze-ice plateau where droplet collection efficiency effects are dominant, roughness spatial evolution is scalable to different geometries using traditional ice shape scaling parameters such as Weber number and stagnation point collection efficiency. However, more data are needed to ensure universality of the correlations based on different accumulation parameters and different stagnation freezing fractions. 


\section{Acknowledgments}

The efforts reported in this paper were partially supported as part of NASA Collaborative Agreement No. NNX12AB85A. The authors thank Mr. Richard E. Kreeger, Mr. Quentin Schwinn, Dr. Sam Lee and Dr. Andy Broeren for their assistance with the study. Finally, the authors thank Peter Tino of the University of Birmingham, UK whose class notes inspired the initial SOM investigation of ice shapes and who provided many suggestions for improving the roughness characterization approach in the early stages if its development. Any opinions presented in this paper are those of the authors and do not reflect the views of NASA or the United States government.

\section{References}

${ }^{1}$ McClain, S.T., Reed, D., Vargas, M., Kreeger, R.E., and Tsao, J.-C.,, (2014), "Ice roughness in Short Duration SLD Icing Events," Presented at the 6th AIAA Atmospheric and Space Environments Conference, June 16-20, Atlanta, GA, AIAA-20142330.

${ }^{2}$ McClain, S.T., Vargas, M., Kreeger, R.E., and Tsao, J.-C., (2015), “A Reevaluation of Appendix C Ice Roughness Using Laser Scanning," SAE 2015 International Conference on Icing of Aircraft, Engines, and Structures, June 22-25, Prague, Czech Republic, SAE2015-01-2098.

${ }^{3}$ Shin, J.,(1994), "Characteristics of Surface Roughness Associated With Leading Edge Ice Accretion,” NASA TM-106459.

${ }^{4}$ Anderson, D. N., and Shin, J., (1997), “Characterization of Ice Roughness from Simulated Icing Encounters,” NASA TM107400

${ }^{5}$ Anderson, D. N., Hentschel, D. B., and Ruff, G. A., (1998), "Measurement and Correlation of Ice Accretion Roughness," NASA CR-2003-211823

${ }^{6}$ Tsao, J.C. and Lee, S., "Evaluation of Icing Scaling on Swept NACA 0012 Airfoil Models," NASA/CR-2012-217419, May 2012.

${ }^{7}$ Tsao, J.C. and Kreeger, R.E., "Experimental Evaluation of Stagnation Point Collection Efficiency of the NACA 0012 Swept Wing Tip," AIAA-2009-4125 and NASA/TM-2010-216102, March 2010.

${ }^{8}$ Langmuir, I. and Blodgett, K. B. "A Mathematical Investigation of Water Droplet Trajectories," Army Air Forces Technical Report No. 5418, February 1946.

9"14 CFR, Aeronautics and Space, Part 25 Airworthiness Standards: Transport Category Airplanes, Section C25.1: Appendix C;" published by the Office of the Federal Register, National Archives and Records Administration, Washington, DC., Amdt. 25140, effective 5-Jan-2015.

10“'14 CFR Parts 25 and 33: Airplane and Engine Certification Requirements in Supercooled Large Drop, Mixed Phase, and Ice Crystal Icing Conditions," (2010), Federal Register, Vol. 75, No. 124, June 29, 37311-37339.

${ }^{11}$ McClain, S. T. and Kreeger, R. E., (2013), “Assessment of Ice Shape Roughness Using a Self-Organizing Map Approach,” Presented at the 5th AIAA Atmospheric and Space Environments Conference, June 24-27, San Diego, CA, AIAA-2013-2546.

${ }^{12}$ Kohonen, T., (2001), Self-Organizing Maps, Berlin: Springer-Verlag, 3rd ed.

${ }^{13}$ McClain, S.T., (2016), "Manual Point Cloud Registration for Combined Ice Roughness and Ice Thickness Measurements," Submitted for presentation at AIAA Aviation 2016, 13-17 June, Washington, D.C.

${ }^{14}$ Wright, W., "User's Manual for LEWICE Version 3.2,” NASA/CR_2008-214255, Nov. 2008. 